http:/ / dx.doi.org/10.21707/gs.v11.n02a05

\title{
Pau-D’arco-Roxo (Handroanthus impetiginosus (MART. EX DC.) MATTOS): CONHECIMENTO E USO MADEIREIRO EM COMUNIDADES RURAIS DO NORDESTE DO BRASIL
}

\section{Irineu Campêlo da Fonseca Filho ${ }^{1 *}$; Brunna Laryelle Silva Bomfim²; Juliana Cardozo de Farias ${ }^{3}$; Fábio José Vieira ${ }^{4}$; Roseli Farias Melo de Barros ${ }^{5}$}

\author{
${ }^{1}$ Mestre em Desenvolvimento e Meio Ambiente - PRODEMA - UFPI; Docente do Instituto Federal de Educação, Ciência e Tecnologia do Piaui- IFPI, \\ campus Angical, Rua Nascimento, 746, Centro, CEP: 64.410-000, Angical do Piaui, Brasil. \\ ${ }^{2}$ Mestre em Desenvolvimento e Meio Ambiente - PRODEMA - UFPI; Docente do Instituto Federal de Educação, Ciência e Tecnologia do Piaui- IFPI, \\ campus Urucuí. \\ ${ }^{3}$ Mestre em Desenvolvimento e Meio Ambiente - PRODEMA - UFPI. \\ ${ }^{4}$ Doutor em Desenvolvimento e Meio Ambiente e Professor da Universidade Estadual do Piaui -UESPI, Campus Professor Barros Araújo, Picos - PI. \\ ${ }^{5}$ Docente do Departamento de Biologia, do mestrado e doutorado em Desenvolvimento e Meio Ambiente da Universidade Federal do Piaui - UFPI, Av. \\ Universitária, 1310, Ininga. CEP: 64049-550. Teresina-PI. \\ *Autorpara correspondência: irineu.campelo@ifpi.edu.br
}

Recebido em 21 de abril de 2016. Aceito em 05 de maio de 2017. Publicado em 29 de julho de 2017.

REsumo - Diversas espécies de plantas lenhosas vêm apresentando declínio de suas populações devido à exploração preferencial de madeira, que se configura como a forma mais danosa para essas populações. Este estudo teve como objetivo investigar os usos e conhecimentos referentes aos recursos de madeira de "pau-d'arco-roxo" (Handroanthus impetiginosus (Mart. Ex DC.) Mattos), espécie amplamente conhecida por seu potencial medicinal, ornamental e madeireiro. Realizaramse entrevistas com auxílio de formulário semiestruturado com todos os chefes de família e/ou responsáveis pela coleta de madeira sobre o conhecimento, preferência e uso da madeira do pau-d'arco, totalizando 28 famílias entrevistadas em duas comunidades do município de Angical do Piauí, no estado do Piauí. Constatou-se que os usos detectados se encaixam nas categorias de construção e tecnologia, não sendo constatado nenhum uso na categoria fitocombustível. A categoria mais citada em ambas as comunidades foi a de construção, devido principalmente ao fato da madeira possuir resistência a xilófagos. A referida planta é amplamente conhecida em ambas às comunidades, sendo que em Novo Horizonte, a mais antiga, apresentou maior diversidade de conhecimento, pois além de maior quantidade de aplicações para a madeira, há um conhecimento mais aprofundado sobre os usos atribuídos à espécie. As informações apresentadas fornecem subsídios para melhor compreensão dos padrões de consumo dos recursos madeireiros da espécie nas comunidades, podendo contribuir na adoção de futuras alternativas de manejo que visem sua garantia para as gerações posteriores.

Palavras-chave: Etnobiologia; Etnobotânica; Recursos Madeireiros; Constructä; Tecnologia.

Pau-d'arco-roxo (Handroanthus impetiginosus (Mart. ex DC.) Mattos): KNowledge and use of wood in RURAL COMMUNITIES IN NORTHEAST BRAZIL

Aвstract - Several species of woody plants have shown a decline in their populations due to the exploitation, especially of wood, which is the most harmful practice to these populations. This study aimed to investigate the uses and knowledge of wood resources of "pau-d'arco-roxo" (Handroanthus impetiginosus (Mart. ex DC.) Mattos), which is widely known for its medicinal, ornamental and timber potential. Semi-structured interviews were carried out with all householders and/or responsible for timber harvesting on the knowledge, preference and use of the pau d'arco wood, totalling 28 interviewed families in two communities in the municipality of Angical do Piauí, in Piauí State, Brazil. The recorded uses were 
classified in the categories of construction and technology, and there were no uses in the phytofuel category. Construction was the most cited category in both communities mainly because the pau d'arco wood is resistant to xylophagous. The studied plant is widely known in both communities, but Novo Horizonte, the older one, showed a higher diversity of knowledge, sincein addition to the high number of the wood uses, there is further knowledge of the uses of the specie. The information shown in this study provides support to a better understanding of patterns of consumption of pau d'arco wood resources in the communities, and may contribute to future management measures to ensure this species for future generations.

Keywords: Ethnobiology; Ethnobotany; Wood Resources; Construction; TeChNology.

Pau-d’arco-roxo (Handroanthus impetiginosus (Mart. ex Dc.) Mattos): conocimiento y uso de la madera en las COMUNIDADES RURALES DEL NORESTE DE BRASIL.

REsumen - Varias especies de plantas leñosas han mostrado una disminución de sus poblaciones debido a suexplotación, especialmente como madera, lo que constituye la práctica más perjudicial para estas poblaciones. El objetivo de este estudio fue investigar los usos y conocimientos de los recursos madereros del "pau-d'arco-roxo" (Handroanthus impetiginosus (Mart. ex DC.) Mattos), que es una especie ampliamente conocida por su potencial medicinal, ornamental y maderero. Se realizaron entrevistas semi-estructuradas, con todos los cabezas de familia y/o responsables por la recogida de la madera, abordando el conocimiento, la preferencia y el uso de la madera del pau-d'arco, totalizando 28 familias entrevistadas en dos comunidades de laciudad de Angical do Piauí, en el estado de Piauí, Brasil. Se observó que los usos registrados se refieren a las categorías de construcción y tecnología y no se constató ningún uso en la categoría fitocombustible. La construcción fue la categoría más citada en ambas comunidades, debido principalmente al hecho de que la madera tiene resistencia a los xilófagos. La dicha planta es ampliamente conocida en ambas comunidades, y en Novo Horizonte, la más antigua, se observó una mayor diversidadde conocimientos, pues además de un número mayor de usos de la madera, hay un conocimiento más profundo de los usos de la especie. La información que se presenta en este estudio proporciona un soporte para una mejor comprensión de los patrones de consumo de los recursos madereros del "pau- d'arco-roxo" en las comunidades y puede contribuir a la adopción de futuras medidas de manejo que buscan garantizar la existencia de esta especie para las generaciones futuras.

Palabras clave: Etnobiología; Etnobotánica; Recursos Madereros; Construcción; Tecnología.

\section{INTRODUÇÃO}

As investigações etnobotânicas se iniciam com a premissa de que as populações rurais possuem conhecimento diversificado sobre os usos dos vegetais. Esse conhecimento pode oferecer aos cientistas modelos de uso sustentável, baseados no argumento de que essas populações sabem usar e conservar os recursos biológicos (Albuquerque e Andrade, 2002). Levantamentos sobre os usos de recursos vegetais em comunidades se tornam importantes porque a maneira pela qual os seres humanos interagem com o meio vegetal circundante é dependente de sua subsistência e necessidades culturais (Blancas et al., 2013).

Dentre esses diversos usos, tem se destacado o consumo preferencial de madeira para fins domésticos, pois esse tipo de extração tem gerado impactos negativos na biodiversidade das matas. Além da redução da diversidade de espécies lenhosas, principalmente, as de maior grau de utilização e valorização para a comunidade, pode ameaçar a conservação biológica, bem como acarretar em prejuízos à vida local (Medeiros et al., 2011).

Torna-se necessário estudar os critérios de seleção de plantas, já que estes podem exercer grande influência na extração da madeira de ambientes naturais (Tabuti et al., 2011). Assim, reforçam este discurso os 
resultados encontrados por Tabuti (2012) em 12 vilas no subcondado de Balawoli, Uganda, em que algumas das espécies lenhosas de uso prioritário têm apresentado declínios nas suas populações devido à superexploração, principalmente para produção de carvão, combustível e postes de madeira.

Assim, a utilização e a coleta de madeira das florestas têm se tornado assunto de interesse para as políticas de planejamento que visam, entre outras coisas, a conservação das florestas (Pandey, 2012).

Faz-se necessário, portanto, avaliar as formas como as pessoas se apropriam desses recursos, os critérios de seleção e as possíveis variáveis que influenciam no uso das plantas. Essas informações contribuem para identificar padrões locais e definir alternativas de manejo e conservação da biodiversidade que respeitem as especificidades locais (Soldati e Albuquerque, 2010). Nesse sentido, Handroanthus impetiginosus (Mart. ex DC.) Mattos, merece destaque como recurso madeireiro. É um representante da família Bignoniaceae, de porte arbóreo, podendo chegar a alturas de 8 a 20 metros, com característica de planta decídua. Sua ocorrência vai desde o estado do Piauí até o de São Paulo, tanto na floresta pluvial atlântica como na floresta semidecídua (Lorenzi, 2002), conhecida localmente como pau d'arco-roxo ou ipê-roxo.

A referida espécie vem sendo alvo de estudos por seu alto valor econômico, medicinal, e também por causa da diminuição da sua disponibilidade em ambientes naturais (Ettori et al., 1996). A árvore é utilizada na arborização urbana (Pestana, 2011), principalmente pela sua beleza, e também pelo seu potencial de sombreamento, que foi comprovado pela sua concentração de clorofila nas folhas e espectro de absorção de luz (Engel, 1991). A casca da H. impetiginosus é conhecida na medicina popular pela sua utilização no tratamento de diabetes, úlcera e sífilis (Warashina et al., 2006). Em virtude disso, grande parte dos estudos direcionados ao H. impetiginosus está relacionada aos seus potenciais antioxidante, antibiótico, bactericida, antifúngico e cicatrizante (Barbosa Filho, 2004; Antunes, 2006; Budni, 2007; Souza, 2008; Coelho et al., 2010).

Entretanto, além desse potencial medicinal, a referida espécie apresenta uma madeira considerada de lei, de excelente qualidade, boa durabilidade e resistência contra organismos que dela se alimentam (xilófagos), sendo difícil de serrar ou pregar (Lorenzi, 2002). Estudos que avaliem a utilização tradicional desse recurso madeireiro ainda são escassos.

Diante disso, selecionou-se essa espécie de grande importância local no fornecimento de recursos madeireiros, com o objetivo de analisar como a população a utiliza, por meio da investigação sobre o conhecimento e o uso de recursos madeireiros provenientes de H. impetiginosus em duas comunidades rurais do município de Angical do Piauí, visando identificar a categoria de uso madeireiro mais importante, comparar o conhecimento nas duas comunidades para melhor compreender as variáveis que influenciam nesse conhecimento, fornecendo subsídios para a adoção de alternativas de manejo para a conservação desse recurso tão importante.

\section{Materiais e métodos}

Área de estudo

O presente estudo foi realizado no município de Angical do Piauí, que está localizado na microrregião do Médio Parnaíba Piauiense (Figura 1), compreendendo uma área de $211 \mathrm{~km}^{2}$, tendo como limites ao norte os municípios de Palmeirais e São Pedro do Piauí, ao sul Amarante, Regeneração, a leste São Gonçalo, Santo Antônio dos Milagres e Jardim do Mulato, e a oeste Palmeirais e Amarante. A sede municipal está localizada nas 
coordenadas geográficas de $06^{\circ} 05^{\prime} 09^{\prime}$ S e $42^{\circ} 44^{\prime} 20^{\prime \prime}$ W e dista cerca de 129 km de Teresina (Aguiar e Gomes, 2004).

O município foi criado pela Lei no 1.054 de 24/07/1954 e possui cerca de 6.672 habitantes e densidade demográfica de 29,86 hab $/ \mathrm{km}^{2}$, onde $21,85 \%$ das pessoas estão na zona rural (IBGE, 2010).

As condições climáticas do município de Angical do Piauí (com altitude da sede a $192 \mathrm{~m}$ acima do nível do mar) apresentam temperaturas mínimas de $20^{\circ} \mathrm{C}$ e máximas de $30^{\circ} \mathrm{C}$, com clima quente tropical. A precipitação pluviométrica média anual registra $1.200 \mathrm{~mm}$, na sede do município, sendo definida no Regime Equatorial Continental, com isoietas anuais entre 800 a $1.400 \mathrm{~mm}$, cerca de cinco a seis meses como os mais chuvosos e período restante do ano de estação seca. Os solos da região são provenientes da alteração de arenitos, siltitos, folhelhos, silexitos, calcários e argilitos. Compreendem solos litólicos, álicos e distróficos, de textura média, pouco desenvolvidos, rasos a muito rasos, fase pedregosa, com floresta caducifólia e/ou floresta sub-caducifólia/ cerrado (Aguiar e Gomes, 2004). Associados ocorrem solos podzólicos vermelho-amarelos, textura média a argilosa, fase pedregosa e não pedregosa, com misturas e transições vegetais de floresta subcaducifólia/caatinga. Secundariamente, ocorrem areias quartzosas, que compreendem solos arenosos essencialmente quartzosos, profundos, drenados, desprovidos de minerais primários, de baixa fertilidade, com transições vegetais, fase caatinga hiperxerófila e/ou cerrado subcaducifólio/floresta subcaducifólia (Aguiar e Gomes, 2004).

As comunidades rurais escolhidas para o estudo foram Assentamento Chapada do Hamilton e Novo Horizonte. Os critérios para escolha foram quantidade de famílias residentes, distância do centro urbano e tempo de fundação.

Figura 1: Mapa de localização do município de Angical do Piauí/PI.
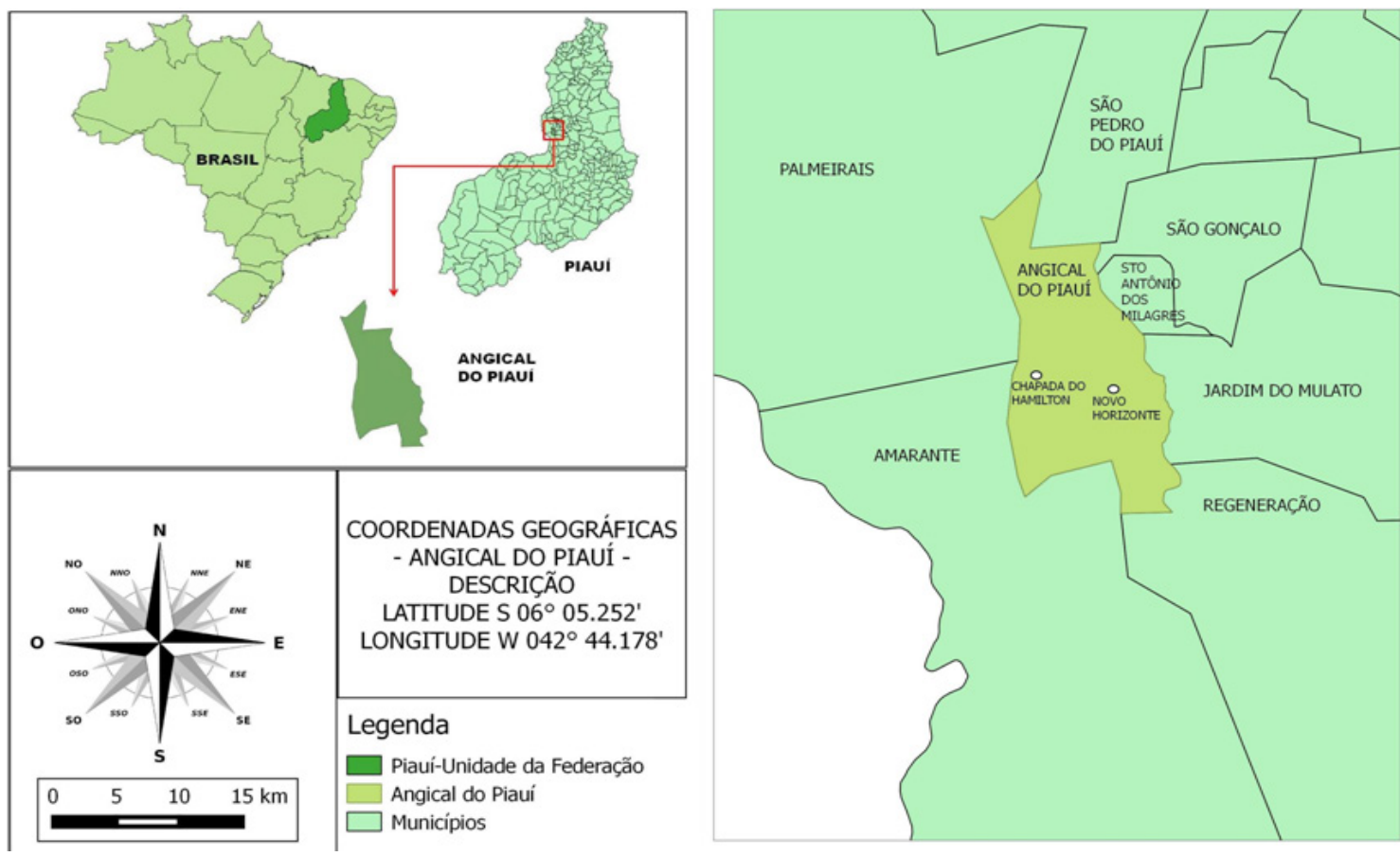

Fonte: IBGE (2015) modificado pelo autor.

O assentamento Chapada do Hamilton é um dos mais recentes de Angical do Piauí, dista 18 km do centro municipal, fundado em 2002 por meio do Projeto de Combate à Pobreza Rural (PCPR), cujo objetivo era contribuir para o alívio da pobreza rural e contemplava inicialmente 19 famílias. No momento da realização da 
pesquisa, apenas 12 famílias residiam na comunidade. Essa evasão foi motivada, segundo os moradores, devido a falta de estrutura, baixa fertilidade da terra e carência de auxílio por parte das autoridades competentes. Ou seja, os financiadores fornecem o crédito para os assentados adquirirem a terra, mas não disponibilizam a capacitação necessária para melhor utilização dessa terra.

A comunidade Novo Horizonte é uma das mais antigas e dista cerca de $12 \mathrm{~km}$ do centro do município. De acordo com moradores mais antigos, o surgimento da comunidade data da década de 1930, quando era conhecida como "Tabuleiro do Couro". Apenas nos anos 1980, um padre sugeriu a mudança no nome da comunidade para "Novo Horizonte". Inicialmente, possuía 45 famílias residentes, porém no momento da pesquisa, segundo agente de saúde, a comunidade possuía 22 famílias residentes.

\section{Coleta de dados}

Observando-se os aspectos éticos da pesquisa, envolvendo seres humanos (Conselho Nacional de Saúde, Resolução n466/2012), os objetivos do trabalho foram explicados à comunidade, em seguida foi solicitada a permissão dos mesmos, através da assinatura do Termo de Consentimento Livre e Esclarecido. O presente trabalho foi submetido à apreciação ética em Comitê de Ética em Pesquisa com o CAAE: 42826815.2.0000.5214, sendo considerado aprovado com o parecer de número: 1.028.579.

Nos primeiros meses de pesquisa, foi adotada a técnica do “rapport” (Barbosa, 2007), em que o pesquisador estabelece contatos prévios com as comunidades com a finalidade de familiarização e criação de um vínculo de confiança com os membros das comunidades.

Foram realizadas 28 entrevistas (11 residentes no assentamento Chapada do Hamilton e 17 residentes na comunidade Novo Horizonte), utilizando-se formulários semiestruturados (Apolinário, 2006), para obtenção dos dados socioeconômicos dos moradores das comunidades (idade, estado civil, escolaridade, renda, ocupação e número de residentes) e informações sobre o uso, conhecimento, preferências e coleta de recursos madeireiros da espécie conhecida localmente como "pau-d'arco-roxo".

$\mathrm{Na}$ comunidade Chapada do Hamilton, todos os 11 domicílios ocupados foram visitados. Na comunidade Novo Horizonte, das 22 residências ocupadas, 17 foram visitadas, as outros quatro não participaram da pesquisa devido à ausência de morador no momento das visitas de campo. Nas residências visitadas, foi entrevistado o chefe de família e/ou responsável pela coleta da madeira.

Para coleta das informações acima citadas, foram formuladas perguntas do tipo: Quais os usos atribuídos à madeira da planta "pau-d'arco-roxo"? Existe preferência por algum uso? Qual o motivo desta preferência? Que características são levadas em consideração na escolha do uso associado?

Para verificar os usos efetivos no dia a dia foi usada a técnica de inventário in situ (Ramos, Medeiros e Albuquerque, 2010).

A coleta botânica obedeceu às técnicas usuais para estudos de taxonomia vegetal (Mori et al., 1989). Posteriormente, foram realizadas técnicas rotineiras de herborização em laboratório conforme procedimento usual (Fidalgo e Bononi, 1989). A identificação botânica se deu mediante análises morfológicas, comparações com materiais incorporados ao acervo do TEPB, bem como confirmada por especialistas. O material herborizado está incorporado ao TEPB (Herbário Graziela Barroso) da Universidade Federal do Piauí (UFPI). 
Análise de dados

Logo após a coleta de dados, os usos atribuídos e os efetivamente verificados foram agrupados em categorias de uso. Dentro dessas categorias, dividiram-se os usos conhecidos e os usos efetivamente detectados no inventário in situ.

A análise quantitativa do conhecimento dos moradores se deu por meio do cálculo do Nível de Fidelidade proposto por Friedman et al. (1986) e utilizado por Ulysséa et al. (2010), segundo os quais o FL apresenta o grau de correspondência entre as respostas dos entrevistados quanto aos usos citados, tendo $\mathbf{F L}=\mathbf{( I p} / \mathbf{I u}) \mathbf{X}$ 100\%, (em que: $\mathbf{F L}=$ nível de fidelidade; $\mathbf{I} \mathbf{p}=$ número de informantes que citaram o uso principal da espécie; $\mathbf{I u}=$ número total de informantes que citaram a espécie para qualquer finalidade).

Para testar as diferenças no número de usos atribuídos à planta $H$. impetiginosus, entre as comunidades estudadas foi aplicado o teste do qui-quadrado $\left(\chi^{2}\right)$. Os testes foram executados utilizando o software BioEstat 5.0 (Ayres et al. 2007).

\section{RESUltados}

Handroanthus impetiginosus (Mart. ex DC.) Mattos (Figura 2) é uma espécie de uso e conhecimento generalizado no horizonte das comunidades estudadas, pois na Chapada do Hamilton e em Novo Horizonte, $83,33 \%$ e $94,44 \%$, respectivamente, a citaram para algum uso madeireiro.

Figura 2: Indivíduos de pau-d'arco-roxo (Handroanthus impetiginosus (Mart. ex DC.) Mattos) na comunidade Novo Horizonte, Angical do Piauí/PI.

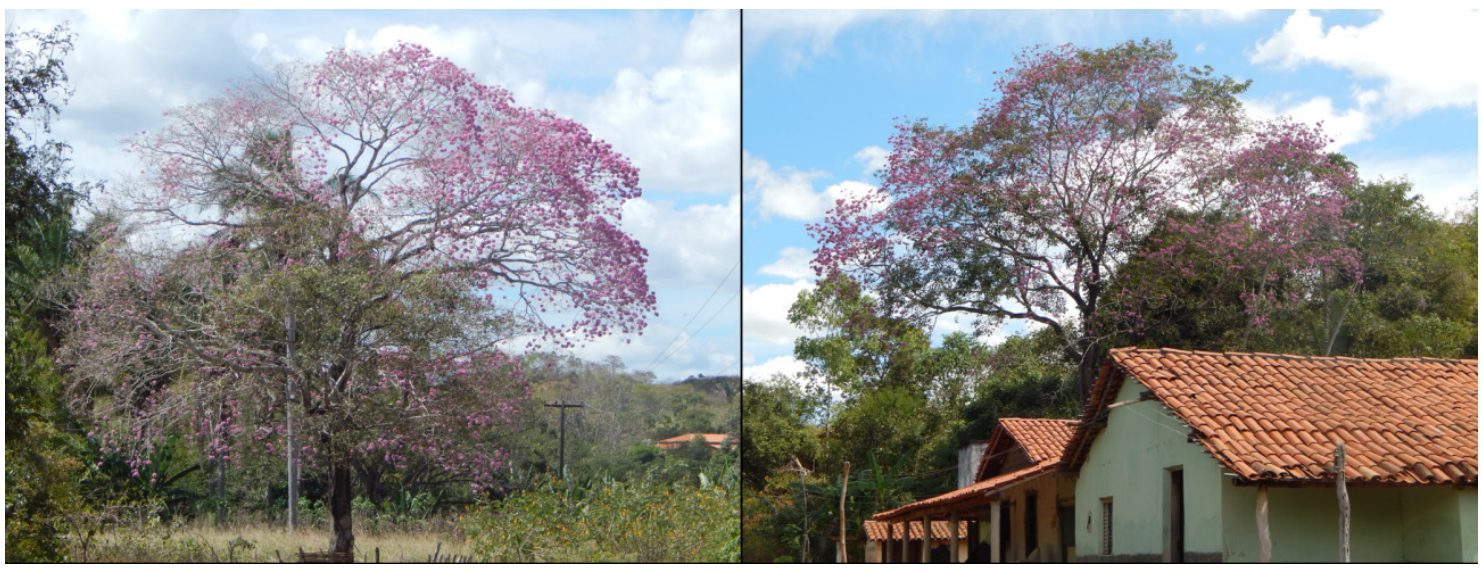

Fonte: arquivo pessoal.

Os usos madeireiros para fins domésticos foram divididos em três categorias de usos principais: construção, tecnologia e fitocombustível. A categoria construção abrange usos como: construção de casas, cercas e currais. A categoria tecnologia está relacionada à confecção de ferramentas, artefatos, móveis e prateleiras para acomodação de panelas (jiraus) e a categoria fitocombustível abrange o uso de carvão e lenha para fornecimento de energia. Em ambas as comunidades, as categorias de uso registradas foram construção e tecnologia (Figura 3). Segundo os entrevistados, a motivação para a ausência de usos de H. impetiginosus para carvão e lenha seria a boa qualidade da madeira da referida espécie, configurando, portanto, um desperdício, na visão dos entrevistados, utilizá-la para esses fins. 
Figura 3: Principais usos de madeiras nas comunidades Chapada do Hamilton e Novo Horizonte, Angical do Piauí/PI. A- Tecnologia. B- Construção.

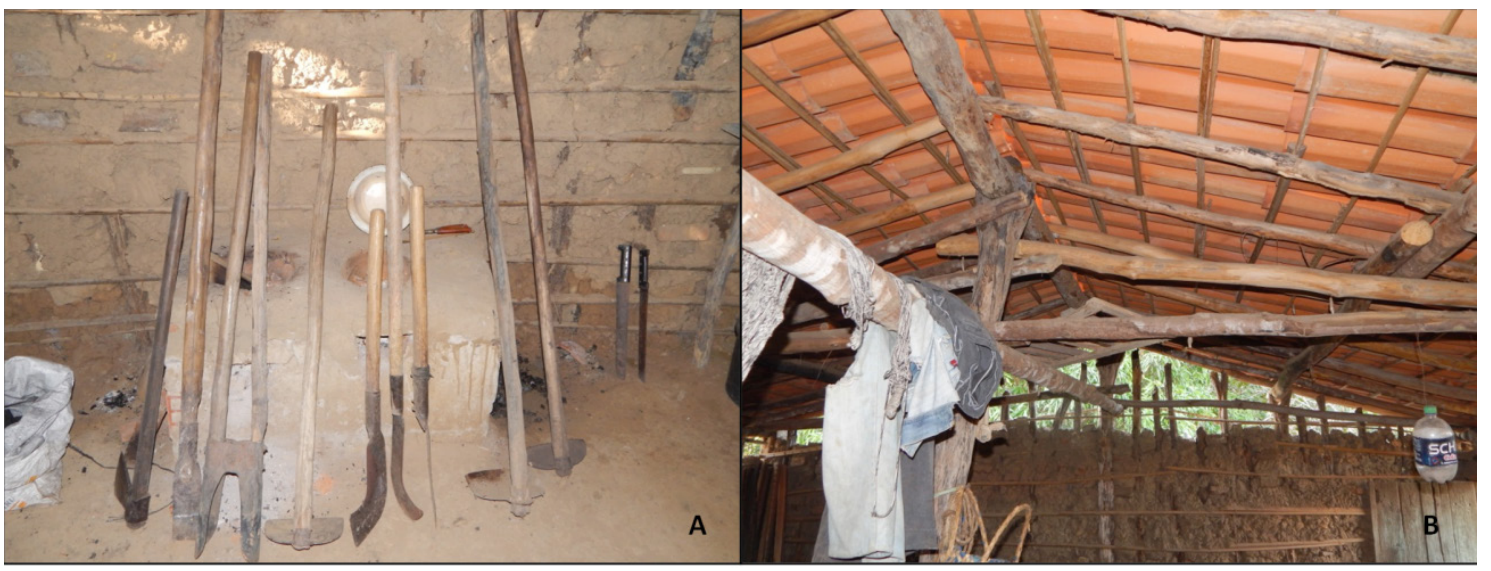

Fonte: arquivo pessoal.

\section{Chapada do Hamilton}

Todos os atores sociais entrevistados que lembraram de H. impetiginosus, citaram pelo menos um uso na categoria construção, configurando-se a categoria mais lembrada. Usos tecnológicos foram lembrados por 70\% dos entrevistados que citaram o pau-d'arco.

Os usos citados para categoria construção estão restritos à confecção de telhados, e na categoria tecnologia, restringe-se à utilização como cabos de ferramentas agrícolas. Quanto à diversidade de uso dentro das categorias, construção apresenta maior diversidade de usos em relação à categoria tecnologia, pois a espécie foi citada com três utilizações específicas na categoria construção, e na categoria tecnologia, foram citadas duas utilizações específicas, como pode ser visto nas Tabelas 1 e 2 .

Tabela 1: Conhecimento, preferência e uso de Handroanthus impetiginosus (Mart. ex DC.) Mattos na categoria construção segundo os moradores da comunidade Chapada do Hamilton, Angical do Piauí/PI

\begin{tabular}{lcccc}
\hline Usos & Conhece & Prefere & Usa & FL \\
\hline Linha & 12 & 10 & 2 & 0,75 \\
Caibro & 3 & - & - & 0,18 \\
Ripa & 1 & 1 & - & 0,06 \\
Total de citações de conhecimento & $\mathbf{1 6}$ \\
\hline
\end{tabular}

Legenda: FL: Nível de Fidelidade. Fonte: dados da pesquisa.

Tabela 2: Conhecimento, preferência e uso da Handroanthus impetiginosus (Mart. ex DC.) Mattos na categoria tecnologia segundo os moradores da comunidade Chapada do Hamilton, Angical do Piauí/PI

\begin{tabular}{lcccc}
\hline Usos - Cabo de ferramenta & Conhece & Prefere & Usa & FL \\
\hline Roçadeira & 7 & 6 & 4 & 0,53 \\
Machado & 6 & 2 & 2 & 0,46 \\
Total de citações de conhecimento & & & $\mathbf{1 3}$ \\
\hline
\end{tabular}

Legenda: FL: Nível de Fidelidade. Fonte: dados da pesquisa. 
De acordo com os informantes, a justificativa para a predominância de usos na confecção de telhados dentro da categoria construção se dá por considerarem que o pau-d'arco-roxo como “madeira pro ar" (Sr. A, 34 anos), no sentido de que tal madeira não é considerada boa para se colocar no solo por se deteriorar rapidamente. As características de interesse citadas para a espécie na categoria construção estão sintetizadas na Tabela 3, onde pode ser visto que a característica de interesse mais lembrada pelos entrevistados foi a resistência a pragas, como os cupins $(62,5 \%)$.

Tabela 3: Características de interesse de Handroanthus impetiginosus (Mart. ex DC.) Mattos na categoria construção segundo os moradores da comunidade Chapada do Hamilton, Angical do Piauí/PI

\begin{tabular}{lc}
\hline Características de interesse & Citações \\
Cupim não come & $62,5 \%$ \\
Não entorta & $19 \%$ \\
Dura mais & $19 \%$ \\
\hline
\end{tabular}

Fonte: dados da pesquisa.

$\mathrm{Na}$ categoria tecnologia, a característica de interesse predominante é a resistência aos serviços mais exigentes e a durabilidade da madeira, justificando a utilização da madeira da espécie para ferramentas como roçadeira e cabo de machado, que segundo eles exigem maior resistência e durabilidade, não sendo utilizada na confecção de outras ferramentas visualizadas no levantamento in situ, como enxada, cavador, semeador, dentre outros, devido à essas ferramentas não exigirem as características supracitadas. Dessa forma, os entrevistados acabam escolhendo uma espécie que fornece madeira mais leve, evitando assim o cansaço precoce no trabalho agrícola. Além de não produzir muito calor no manuseio diário.

Em relação à parte da planta a ser utilizada, a categoria construção se torna mais impactante, pois todos os entrevistados citam o tronco principal como parte da planta utilizada. Na categoria tecnologia, todos os entrevistados afirmam que o material utilizado para a confecção dos cabos das ferramentas é proveniente dos ramos da planta.

\section{Novo Horizonte}

A categoria de uso mais citada pelos entrevistados na comunidade Novo Horizonte também foi a categoria construção, citada por $94,12 \%$ dos entrevistados, enquanto a categoria tecnologia foi lembrada por $76,47 \%$ dos informantes.

A comunidade apresentou maior diversidade de usos para a espécie H. impetiginosus na categoria construção, num total de seis utilizações específicas, como pode ser visualizado na Tabela 4.

Nessa comunidade, além de ser registrada maior diversidade de usos na categoria construção, foram registrados usos de H. impetiginosus em contato com o solo (como forquilha ${ }^{1}$ ), fato não constatado na comunidade anterior. Tal uso foi justificado por um informante nas seguintes palavras: "o pau-d'arco maduro possui miolo, não estraga no chão" (Sr. A., 34 anos).

1 Madeira em formato de "Y" que dá suporte, geralmente suspendendo algo. 
Tabela 4: Conhecimento, preferência e uso de Handroanthus impetiginosus (Mart. ex DC.) Mattos na categoria construção segundo os moradores da comunidade Novo Horizonte, Angical do Piauí/PI

\begin{tabular}{lcccc}
\hline Usos & Conhece & Prefere & Usa & FL \\
Linha & 14 & 8 & 4 & 0,53 \\
Caibro & 6 & 2 & 1 & 0,23 \\
Viga & 2 & - & 2 & 0,07 \\
Forquilha & 1 & - & 1 & 0,03 \\
Travessa & 2 & 1 & 2 & 0,07 \\
Armador & 1 & - & 1 & 0,03 \\
Total de citações de conhecimento & & $\mathbf{2 6}$ \\
\hline
\end{tabular}

Legenda: FL: Nível de Fidelidade. Fonte: dados da pesquisa.

$\mathrm{Na}$ categoria tecnologia, também foi apresentado maior diversidade de uso em relação ao registrado na comunidade anterior, com um total de quatro utilizações específicas na categoria conforme Tabela 5.

Tabela 5: Conhecimento, preferência e uso de Handroanthus impetiginosus (Mart. ex DC.) Mattos na categoria tecnologia segundo os moradores da comunidade Novo Horizonte, Angical do Piauí/PI. Legenda: FL: Nível de Fidelidade.

\begin{tabular}{lcccc}
\hline Usos & Conhece & Prefere & Usa & FL \\
Cabo de ferramentas & 16 & 10 & 8 & 0,66 \\
Moldura da porta & 2 & - & 2 & 0,08 \\
Móveis & 3 & 1 & 3 & 0,12 \\
Tábuas & 2 & - & 2 & 0,08 \\
Total de citações de conhecimento & & & $\mathbf{2 3}$ \\
\hline
\end{tabular}

Fonte: dados da pesquisa.

As características de interesse para os usos nas categorias construção e tecnologia estão resumidas nas Tabelas 6 e 7, onde pode se constatar que a resistência a cupins apresenta maior percentual de citação na categoria construção $(57,7 \%)$, e que a resistência apresenta maior percentual em tecnologia $(88,23 \%)$, as mesmas características de interesse que tiveram maior percentual de citação na comunidade Chapada do Hamilton.

Tabela 6: Características de interesse na categoria construção de Handroanthus impetiginosus (Mart. ex DC.) Mattos segundo a comunidade Novo Horizonte, Angical do Piauí/PI.

\begin{tabular}{lc}
\hline Características de interesse & Citações \\
\hline Resistência aos cupins & $57,7 \%$ \\
Durabilidade & $19 \%$ \\
Ramos retilíneos & $11,5 \%$ \\
Tamanho & $11,5 \%$ \\
\hline
\end{tabular}

Fonte: dados da pesquisa.

Em relação à parte da planta utilizada na categoria construção, todos os entrevistados relataram uso do tronco principal. Na categoria tecnologia, 69,56\% das citações apontaram o uso dos ramos da planta, e 30,43\% dos informantes afirmaram utilizar o tronco. 
Tabela 7: Características de interesse na categoria tecnologia de Handroanthus impetiginosus (Mart. ex DC.) Mattos segundo as comunidades Novo Horizonte e Chapada do Hamilton, Angical do Piauí/PI.

\begin{tabular}{lccc}
\hline Características de interesse & F. C. & $\begin{array}{c}\text { Chapada do } \\
\text { Hamilton }\end{array}$ & $\begin{array}{c}\text { Novo } \\
\text { Horizonte }\end{array}$ \\
\hline Resistência & $88,23 \%$ & 14 & 16 \\
Ramos retilíneos & $5,88 \%$ & & 2 \\
Disponibilidade & $2,94 \%$ & & 1 \\
Peso & $2,94 \%$ & & 1 \\
\hline
\end{tabular}

Legenda: F. C. - Frequência de citação. Fonte: dados da pesquisa.

Para o total de usos relatados, e os usos preferidos atribuídos para H. impetiginosus, pode-se observar que a comunidade Novo Horizonte apresenta maior número de utilizações atribuídas ao pau-d'arco-roxo, 11 aplicações diferentes, sendo que na comunidade Chapada do Hamilton apresenta apenas cinco (Figura 4). Entretanto, não houve diferença significativa no número de usos atribuídos $\left(\chi^{2}=2,25 ; \mathrm{p}=0,1336\right)$.

Figura 4: Riqueza e preferência de usos atribuídos para Handroanthus impetiginosus (Mart. ex DC.) Mattos nas comunidades rurais Chapada do Hamilton e Novo Horizonte, Angical do Piauí/PI. $\chi^{2}=$ valor do qui-quadrado; são considerados significativos todos os resultados cujo valor de $\mathrm{p}<0,05$.

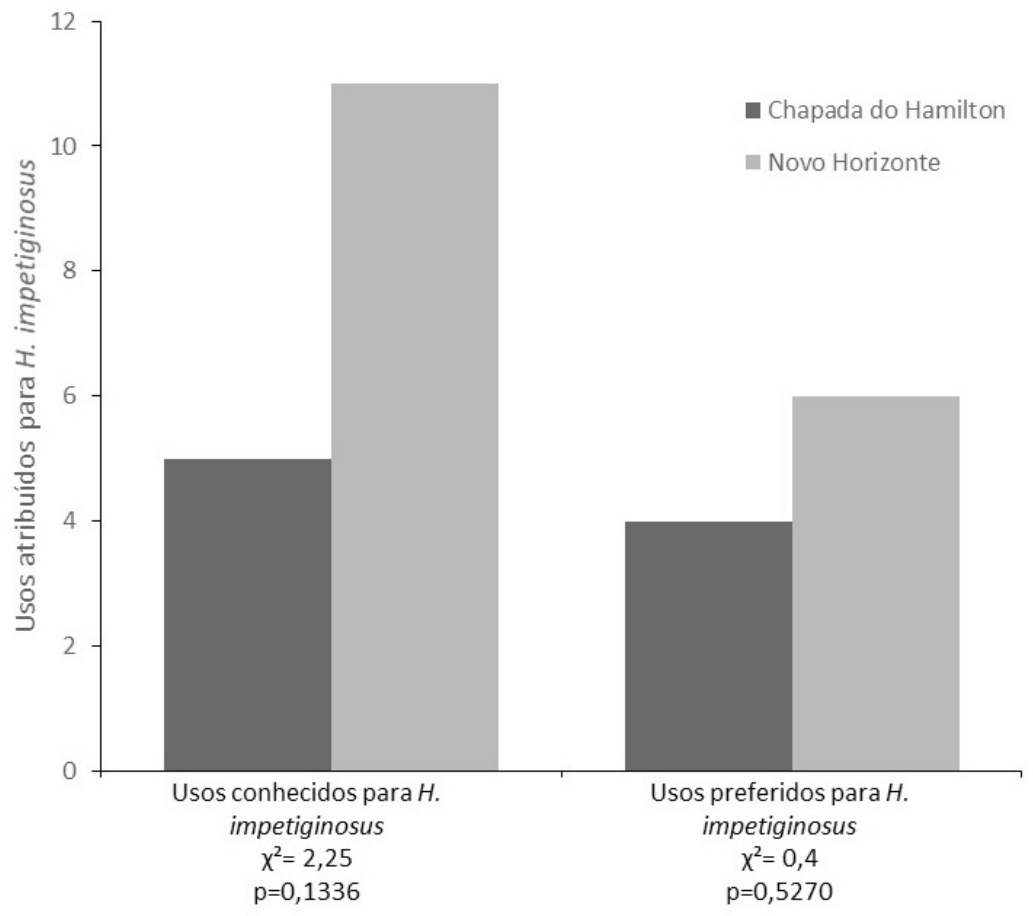

\section{Discussão}

De acordo com os resultados apresentados, não há diferença significativa no conhecimento e na variedade de usos atribuídos à espécie entre as comunidades estudadas, apesar de não ter sido registrada a presença de indivíduos de H. impetiginosus na mata do entorno da comunidade Chapada do Hamilton durante as incursões de campo. Esse padrão não tem sido encontrado em estudos que testam a hipótese da aparência ecológica, 
que relaciona o uso com a disponibilidade do recurso na área, como no estudo de Tunholi (2013), em que 19 espécies apresentaram correlação positiva entre os parâmetros fitossociológicos e o valor de uso.

A comunidade Novo Horizonte, H. impetiginosus apresenta maior versatilidade de usos, sinalizando que pode haver maior pressão de uso, pois como afirmam Ferreira Júnior et al. (2012), é esperado que espécies que possuem alta versatilidade de uso tenderem a apresentar maior incidência de coleta.

Em ambas as comunidades houve consenso em relação à rejeição ao uso de $H$. impetiginosus em contato com o solo, como na confecção de cercas. Esse fato diverge do observado no estudo de Chaves et al. (2014), que constataram que o tipo mais comum de cerca, a de arame farpado, utiliza estacas de sabiá (Mimosa caesalpiniifolia Benth.), em associação com outras espécies, como pau-d'arco-roxo (H. impetiginosus), aroeira (Myracrodruon urundeuva Allemão) ou jatobá (Hymenaea courbaril L.).

Os entrevistados no estudo dividiram o uso geral da madeira em "madeira pro ar" e "madeira pro solo", a qual também foi constatada no trabalho de Lima et al. (2011). Entretanto no presente trabalho, a espécie $H$. impetiginosus foi agrupada pelos moradores no grupo de "madeira pro ar", sendo utilizada principalmente na construção de telhados, e no trabalho de Lima et al. (2011) a espécie foi colocada no grupo de "madeiras pro solo".

Dentre as características de interesse citadas pelos moradores de ambas as comunidades, é que a madeira de H. impetiginosus possui maior resistência aos cupins, tendo assim maior durabilidade. Esse fato foi comprovado em estudos de laboratório desenvolvidos por Paes (2003), que apresentou o pereiro e o pau-d'arco como as espécies mais resistentes ao ataque de cupins, dentre as analisadas, as quais apresentam potencial para serem utilizadas em locais infestados pela espécie de cupim Nasutitermes corniger (Motsch.).

Outro motivo de preferência com alto índice de citação nas comunidades está relacionado à resistência da madeira de H. impetiginosus a fungos, que também foi comprovado em parte por estudos em laboratório de Paes (2005), que constatou que as madeiras de aroeira e braúna (cerne), pereiro e pau-d'arco apresentam resistência aos fungos causadores da podridão-mole que atacam madeiras utilizadas em locais expostos à alta umidade, fato esse que indica que essas madeiras apresentariam melhor desempenho nessas condições que as demais espécies estudadas.

\section{Conclusão}

Handroanthus impetiginosus (Mart. ex DC.) Mattos é uma espécie amplamente conhecida e utilizada no horizonte nas duas comunidades estudadas, possuindo relevante quantidade e diversidade de usos atribuídos à espécie.

Grande parte das características de interesse citadas pelos participantes da pesquisa, como resistência e durabilidade, possui fundamentação na literatura científica.

Não foi constatada diferença significativa no conhecimento e na variedade de usos atribuídos à espécie entre as comunidades estudadas, sugerindo que a idade da comunidade e a distância do centro urbano não influenciaram no conhecimento sobre o uso da madeira dessa espécie. Entretanto, foi averiguada maior versatilidade de usos na comunidade Novo Horizonte, indicando que a espécie pode estar sofrendo maior pressão de uso. 


\section{REFERÊNCIAS}

Aguiar RB e Gomes JRC. 2004. Projeto cadastro de fontes de abastecimento por água subterrânea, estado do Piauí: diagnóstico do município de Angical do Piauí, Fortaleza: CPRM - Serviço Geológico do Brasil. 20p. Albuquerque UP e Andrade LHC. 2002. Conhecimento botânico tradicional e conservação em uma área de caatinga no estado de Pernambuco, Nordeste do Brasil. Acta Botanica Brasilica, 16(3): 273-285.

Albuquerque UP, Lucena RFP, Cunha LVFC e Alves RRN. 2014. Methods and Techniques in Ethnobiology and Ethnoecology. New York: Humana Press, 476p.

Antunes RMP, Lima EO, Pereira MSV, Camara CA, Arruda TA, Catão RMR, Barbosa TP, Nunes XP, Dias CS e Silva TMS. 2006. Atividade antimicrobiana "in vitro" e determinação da concentração inibitória mínina (CIM) de fitoconstituintes e produtos sintéticos sobre bactérias e fungos leveduriformes. Revista Brasileira de Farmacognosia, 16 (4): 517-524.

Apolinário, F. 2006. Metodologia científica. - Filosofia e prática da pesquisa. São Paulo: Thomson Leaming. $240 \mathrm{p}$

Ayres M, Ayres JR., Ayres DL e Santos AS. 2007. BioEstat 5.0 - aplicações nas áreas de ciências biológicas e médicas. Belém: Sociedade Civil Mamirauá. 364 p.

Barbosa AR. 2007. Os humanos e os répteis da mata: uma abordagem etnoecológica de São José da Mata Paraíba. 2007. 123 f. Dissertação (Mestrado em Desenvolvimento e Meio Ambiente) - Universidade Federal da Paraíba, João Pessoa.

Barbosa-Filho JM, Lima AS, Camorim EL, Sena KXF, Almeida JRG, Da-Cunha VL, Silva MS, Agra MF e Braz-Filho R. 2004. Botanical study, phytochemistry and antimicrobial activity of Tabebuia aurea. Phyton, 73: 221-228.

Blancas J, Casas A, Salicrup DP, Caballero J, Veja, E. 2013. Ecological and sóciocultural factors influencing plant management in Náhuatl communitie of the Tehuacán Valley Mexico. Journal of Ethnobiology and Ethnomedicine, 9 (39): 1-13.

Budni P, Petronilho FC, Citadini-Zanette V, Marcondes C, Zoch AN, Reginatto FH e Dal-Pizzol F. 2007. Estudos preliminares da atividade antioxidante do extrato hidroetanólico de folhas jovens e adultas de Tabebuia heptaphylla (Vell.) Toledo (ipê-roxo). Latin American Journal Pharmacy, 26 ( 3): 394-398.

Chaves EMF, Chaves EBF, Servio Junior EM e Barros RFM. 2014). Conhecimento tradicional: a cultura das cercas de madeira no Piauí, Nordeste do Brasil. Etnobiología, 12: 31-43.

Coelho JM, Antoniolli AB, Silva DN, Carvalho TMMB, Pontes ERJC e Odashiro AN. 2010. O efeito da sulfadiazina de prata, extrato de ipê-roxo e extrato de barbatimão na cicatrização de feridas cutâneas em ratos. Revista do Colégio Brasileiro de Cirurgiões, 37 (1): 45-51. 
Conselho Nacional de Saúde. 2013. Resolução no 466, de 12 de Dezembro de 2012. Estabelece as diretrizes e normas brasileiras regulamentadoras de pesquisas envolvendo seres humanos. Diário Oficial da União. $n^{\circ}$ 12, quinta-feira, 13 de junho de 2013, Seção 1, Pág. 59. Disponível em: http://conselho.saude.gov.br/ resolucoes/2012/Reso466.pdf Acesso em 04 janeiro 2015.

Cronquist, A. 1981. An integrated system of classification of flowering plants. New York: Columbia University Press, 1981. 1262p.

Dahlgren RMT e Clifford HT. 1980. The monocotyledones: a comparative study. Academic Press, London.

Engel VL e Poggiani F. 1991. Estudo da concentração de clorofila nas folhas e seu espectro de absorção de luz em função do sombreamento em mudas de quatro espécies florestais nativas”. Revista Brasileira de Fisiologia Vegetal, 3 (1): 39-45.

Ettori LC, Siqueira ACMF, Sato AS e Campos OR. 1996. Variabilidade genética em populações de ipê-roxo Tabebuia heptaphylla (Vell.) Tol. - para conservação ex situ”. Revista do Instituto Florestal, 8 (1): 61-70.

Ferreira Júnior WS, Siqueira CFQ e Albuquerque UP. 2012. Plant stem bark extractivism in the Northeast semiarid region of Brazil: A new aport to utilitarian redundancy model. Evidence-Based Complementary and Alternative Medicine, 1-11.

Fidalgo O e Bononi VLR. 1989. Técnicas de coleta, preservação e herborização de material botânico. Instituto Botânica. São Paulo.

Friedman J, Yaniv Z, Dafni A e Palewith D. 1986. A preliminar classification of the healing potencial of medicinal plants, based on a rational analysis of na ethnopharmacological field survey among bedouins in the Negev desert, Israel. Journal of Ethnopharmacology, 16: 275-287.

Grandis A, Aleixo AM, Dédalo MFN e Ruggiero AC. 2006. Avaliação da capacidade antioxidante do extrato de pau-d' arco (Tabebuia avellanedae) e suas frações. Anais da 58 Reunião Anual da SBPC - Florianópolis.

Handroanthus impetiginosus (Mart. ex DC.) Mattos. 2012. The Plant List. (2012 [last update]). Citado em 27 Julho de 2015. Disponivel em World Wide Web: http//:www.theplantlist.org

Lima JS, Oliveira DM, Nascimento Júnior JEN, Mann RS e Gomes LJ. 2011. Saberes e uso da flora madeireira por especialistas populares do agreste de Sergipe. Sitientibus Série Ciências Biológicas, 11 (2): 239-53.

Lorenzi, Harri. 2002. Árvores brasileiras: manual de identificação e cultivo de plantas arbóreas do Brasil. v. 1, 4 ed. Instituto Plantarum, Nova Odessa, SP.

Medeiros PM, Silva TC, Almeida ALS e Albuquerque UP. 2011. Pressure Indicators of Wood Resource Use in an Atlantic Forest Area, Northeastern Brazil. Environmental Management, 47: 410-424.

Oswald EH. 1993/94. "Lapacho", British Journal of Phytotherapy, 3 ( 3): 112-117.

Paes JB, Morais VM, Farias Sobrinho DW e Bakke OA. 2003. Resistência natural de nove madeiras do semiárido 
brasileiro a cupins subterrâneos, em ensaio de laboratório. Cerne, 9 (1): 36-47.

PAES JB, Morais VM e Lima CR. 2005. Resistência natural de nove madeiras do semi-árido brasileiro a fungos causadores da podridão-mole. Revista Árvore, 29 (3): 365-371.

Pandey R. 2012. Domestic Burning of Fuelwood in a Subsistence Tribal Economy of Lower Himalayas, India: Some Implications Based on Exploratory Analysis. Small-scale Forestry, 11 (1): 119-130.

Pestana LTC, Alves FM e Sartori ALB. 2011. Espécies arbóreas da arborização urbana do centro do município de Campo Grande, Mato Grosso do Sul, Brasil. REVSBAU, 6 (3): 1-21.

Ramos MA, Medeiros PM e Albuquerque UP. 2010. Métodos e técnicas aplicados a estudos etnobotânicos com recursos madeireiros. In: Albuquerque UP, Cunha LVC, Lucena RFP e Alves R R N. (Eds) Métodos e técnicas na pesquisa etnobiológica e etnoecológica, Recife: NUPEEA, p.329-350.

Soldati GT e Albuquerque UP. 2010. Produtos Florestais não Madeireiros: uma visão geral. In: Albuquerque UP e Hanazaki N. (Org.). Árvores de valor e o valor das árvores: pontos de conexão. Recife: NUPEEA, p. 17-59.

Souza MAAD, Silva ARD, Ferreira MA, Lemos MJD, Ramos RG, Ferreira ABB e Souza SRD. 2008. Atividade biológica do lapachol e de alguns derivados sobre o desenvolvimento fúngico e em germinação de sementes. Química Nova, 31 ( 7): 1670-1672.

Tabuti JRS, Muwanika VB, Arinaitwe MZ e Ticktin T. 2011. Conservation of priority woody species on farmlands: A case study from Nawaikoke sub-county, Uganda. Applied Geography, 31 (2): 456-462.

Tabuti JRS. 2012. Important Woody Plant Species, their Management and Conservation Status in Balawoli Subcounty, Uganda. Ethnobotany Research \& Applications, 10: 269-286.

Tunholi VP, Ramos MA e Scariot A. 2013. Availability and use of woody plants in a agrarian reform settlement in the cerrado of the state of Goiás, Brazil. Acta Botanica Brasilica, 27 (3): 604-612.

Ulysséa MA, Hanazaki N e Lopes BC. 2010. Percepção e uso dos insetos pelos moradores da comunidade do Ribeirão da Ilha, Santa Catarina, Brasil. Revista Biotemas, 23: 191-202.

Warashina T, Nagatani Y e Noro T. 2006. Constituents from the bark of Tabebuia impetiginosa. Chemical and Pharmaceutical Bulletin, 54 (1): 14-20. 